\title{
Toward a Predictive Atomistic Model of Ion Implantation and Dopant Diffusion in Silicon
}

\author{
M.-J. Caturla \\ M. D. Johnson \\ J. Zhu
}

September 8, 1998

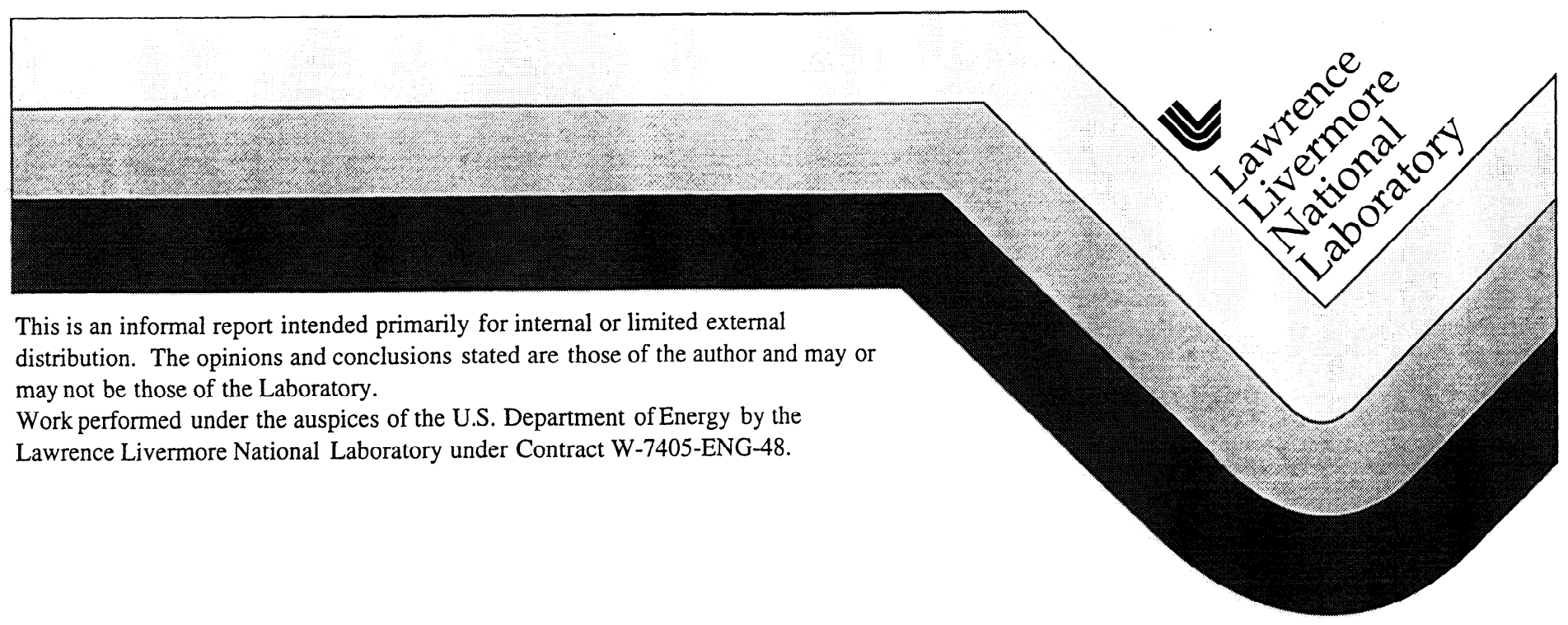




\section{DISCLAIMER}

This document was prepared as an account of work sponsored by an agency of the United States Government. Neither the United States Government nor the University of California nor any of their employees, makes any warranty, express or implied, or assumes any legal liability or responsibility for the accuracy, completeness, or usefulness of any information, apparatus, product, or process disclosed, or represents that its use would not infringe privately owned rights. Reference herein to any specific commercial product, process, or service by trade name, trademark, manufacturer, or otherwise, does not necessarily constitute or imply its endorsement, recommendation, or favoring by the United States Government or the University of California. The views and opinions of authors expressed herein do not necessarily state or reflect those of the United States Government or the University of California, and shall not be used for advertising or product endorsement purposes. 


\title{
Toward a predictive atomistic model of ion implantation and dopant diffusion in Silicon
}

\author{
M.-J. Caturla, M. D. Johnson, J. Zhu \\ Lawrence Livermore National Laboratory, Livermore CA-94550
}

\begin{abstract}
We review the development and application of kinetic Monte Carlo simulations to investigate defect and dopant diffusion in ion implanted silicon. In these type of Monte Carlo models, defects and dopants are treated at the atomic scale, and move according to reaction rates given as input parameters. These input parameters can be obtained from first principles calculations and/or empirical molecular dynamics simulations, or can be extracted from fits to experimental data. Time and length scales differing several orders of magnitude can be followed with this method, allowing for direct comparison with experiments. The different approaches are explained and some results presented. ${ }^{1}$
\end{abstract}




\section{A. Introduction}

The microscopic structure of defects and dopants in semiconductors has been the subject of many theoretical [1-4] and experimental [5-9] studies over the last three decades Regarding theory, the development in the last few years of powerful computers has enabled the application of ab initio calculations based on density functional theory within the local density approximation, and of large scale molecular dynamics simulations (MD). MD simulations have been used extensively to study the defect production in irradiated semiconductors, such as Silicon [10-12] and $\mathrm{SiC}$ [13] . These calculations provide a quantitative understanding of many fundamental phenomena such as, for example, defect formation and migration energies. . Even more interestingly, the interaction between defects and impurities has also been studied in detail using these models, and binding energies between different defect and dopant structures have been obtained[3, 14]. These values provide the basis for understanding how clustering, precipitation and trapping of defects and impurities occurs. Other more approximate models such as tight binding and classical molecular dynamics have also been used to study defect diffusion and binding energies of clusters of defects in Silicon [15-17]. These models have the advantage of being computationally more efficient, therefore allowing for dynamic studies of defect migration, as well as computation of larger defect structures (clusters of vacancies and interstitials). However, the applicability of these models depends on the reliability of the semi-empirical potentials they are based on.

Concurrently, experimental techniques have been used for the identification of defects in silicon. Electron paramagnetic resonance (EPR) experiments have characterized vacancies in silicon, their different charge states, migration energies and their interactions with impurities (for a review see Watkins [5]). Deep level transient spectroscopy measurements 
are also used to characterize vacancies and interstitials, as well as dopant and impurity complexes, providing values for binding energies of different dopant-defect pairs [18]. Recently, diffuse X-ray scattering (DXS) has been applied to study the small defects created during ion bombardment and damage annealing $[9,19]$. The interpretation of the results obtained by this technique is complicated in the case of semiconductors, due to the similar relaxation volumes for vacancies and self-interstitials, and the possible formation of large disordered or amorphous zones during the collision cascade. However, this technique was able to measure the formation of close Frenkel pairs for irradiation at $4 \mathrm{~K}$, that are not present during room temperature irradiation. These close Frenkel pairs have also been identified by tight binding molecular dynamics simulations [16]. Recent combined efforts between experiments and simulations allow for a more clear interpretation of the experimental results obtained with DXS [20].

Other techniques, such as high resolution electron microscopy (HR-TEM) and scanning tunneling microscopy (STM), allow for direct observation of microscopic structures. Rodlike defects have been identified in silicon using TEM. These defects were produced by silicon self-irradiation and high temperature annealing [6]. The dissolution rate of these \{311\} defects at different temperatures was obtained from these measurements and a link was established between the transient enhanced diffusion observed in Boron and the dissolution of these defects. On the other hand, STM was used to study surface evolution during annealing at different temperatures [7]. These experiments show a decrease in the population of the adatom layer during low temperature annealing and a re-population of this layer when the temperature is increased. These phenomena has been associated to different arrival rates of vacancies and interstitials to the surface, as a consequence of the different stabilities of clusters of vacancies and interstitials. All these novel experiments give a better insight to complicated phenomena such as defect and impurity diffusion, clustering and trapping. Moreover, they can be used to validate some of the theoretical calculations 
discussed above. Nevertheless, a large controversy still exists regarding basic parameters such as migration energies of vacancies and interstitials [21].

Despite these new advances both in experiments and simulations, a time gap still exists between the two in the most common situations. Phenomena such as defect diffusion can not be studied using, for instance, molecular dynamics, since only times on the order of nanoseconds can be followed with this type of simulations. Instead, rate theory is used to model defect and dopant diffusion in semiconductors. In particular, these models have been applied to study transient enhanced diffusion [22-25]. However, in most of the cases, this approach is only valid in close to equilibrium situations. Moreover, up to now, all these models relayed on a set of parameters that had to be fitted in order to reproduce a particular experimental observation, therefore, limiting the predictability of the model.

We will present a simulation model that provides a link between microscopic values, such as migration and binding energies, and macroscopic phenomena. This kinetic Monte Carlo model (k-MC) can simulate time scales differing several orders of magnitude, as well as computational systems on the order of microns. This type of simulations can explore the different paths for defect agglomeration and their results can be directly compared with experiments, both high resolution microscopy and SIMS concentration profiles, without fitting the input parameters. Thus, it provides a physically based model of defect diffusion that, moreover, is not restricted to close to equilibrium situations. A complete consistent set of parameters will constitute a model that is predictive and can be used to study different experimental conditions. Furthermore, the failure to reproduce some of the experiments can be easily understood in terms of the approximations introduced in the model, instead of having to re-fit the input parameters, such as the case of continuum models. The results obtained from these simulations can also be exported into continuum models, such us SUPREME [26] or Alamode [27], and consequently, construct a non-computationally 
demanding, physically based model, that allows for prediction of defect profile evolution for different device conditions.

In the next section we will explain the input necessary for a generic kinetic Monte Carlo simulation of ion implantation and diffusion, and the model will be described. Section 3 describes some results obtained with these model as well as the different approaches. We end up with some conclusions and future developments and applications of this method.

\section{B. The kinetic Monte Carlo simulator}

\section{B.1 General description}

Heinisch and co- workers used for the first time a Monte Carlo model to simulate the migration, agglomeration and dissociation of the defects produced by $25 \mathrm{keV} \mathrm{Cu}$ selfirradiation at different temperatures [28]. This model has been recently implemented in order to simulate implantation and diffusion of defects in Silicon $[29,30]$. We will explain here, in general terms, our implementation of such simulation.

Our model considers point defects (vacancies and silicon self-interstitials), dopants (Boron), impurities (Carbon), extended defects (vacancy clusters, self-interstitial clusters) and mixed clusters (B-I clusters, C-I clusters) as individual objects with a basic set of properties such as size, shape, jump rates and dissociation rates. There is not an underlying lattice in this model, since defects are considered to diffuse by a random walk. The different possible reactions, such as formation or dissociation of clusters, are given as input tables that can be easily modified. The values for dissociation rates of clusters and jumping rates of defects and dopants are given by the binding and migration energies, respectively, that can be obtained by different methods as we will describe below. A jump distance equal to first nearest neighbors $(0.235 \mathrm{~nm})$ has been considered in our simulations. The initial 
stage of the simulation consists of the three dimensional distribution of point defects, impurities and dopants. Events are picked according to the reaction rates and the number of objects of one type. The object picked is either placed into a new location (in case of a migration event, or dissociation from a cluster), removed from the system (in case of recombination) or changed into another object (in case of agglomeration in a cluster). The former and new neighbors of the object picked must be also modified. This step is the most computationally intensive in the simulation. The time is incremented after each event considering the total reaction rates. A kinetic Monte Carlo simulation, therefore, includes a microscopic description of defects and impurities. However, due to the simple description of a complex system, such as a doped silicon lattice, times and length scales ranging several orders of magnitude can be followed by this method.

\section{B.2 The input data for a k-MC simulation}

The input data necessary for a basic simulation of defect diffusion consists of the jump rates for vacancy and self-interstitial migration, or, in other words, the diffusivity at different temperatures. In the case of Silicon, values for diffusivities of vacancies and selfinterstitials exist in the literature and have been calculated using Stillinger-Weber molecular dynamics [15] and tight binding molecular dynamics [17]. Experimentally these diffusivities are not known, since only the product of defect concentration and defect diffusivity can be obtained, and high controversy still exists regarding the independent values [21, 31]. Migration energies of vacancies and interstitials have also been obtained using first principles local density approximation (LDA) calculations [3], however, these type of calculations, although the most accurate, do not provide the diffusivity pre-factor necessary to calculate the different jump rates, since they are all performed at zero temperature. 
For systems involving high defect concentrations, it is necessary to include in the Monte Carlo simulation the possibility of defect clustering, and, therefore, the binding energy of vacancy clusters and self-interstitial clusters must be obtained. The binding energy will provide the rate of dissolution of the clusters at different temperatures. Values for binding energies of vacancies have been calculated using empirical molecular dynamics with different interatomic potentials, such as the Stillinger-Weber potential [15] and the Tersoff potential [17]. Tight binding molecular dynamics has also been used to calculate vacancy binding energies [32]. Silicon self-interstitial binding energies have only been calculated using Stillinger-Weber molecular dynamics for very small clusters (up to size 5) [15]. These calculations seem to indicate that the interstitial binding energies are larger than those of the vacancy clusters. This difference plays a very important role in the later evolution of the vacancy and interstitial clustering and it will influence dramatically the outcome of the kinetic Monte Carlo simulation. Therefore, the study of the binding energies of interstitial clusters and their evolution to rod-like defects is of great importance for accurate modeling of defect and dopant diffusion.

The simulation of dopant diffusion requires, primarily of the study of the migration path of the dopant of interest. The path and energies for Boron migration in Silicon has been obtained using (LDA) calculations both for neutral species [3] and charged species [2, 33]. Other Silicon dopants, such as Arsenic, have also been studied using these method [4, 35]. The migration path for dopants will impose an important difference in the type of Monte Carlo calculation used. In the case of Boron, the migration is through a 'kick out' mechanism, with the aid of silicon self-interstitials. Boron atoms will migrate by jumping to an interstitial position. This allows for a Monte Carlo calculation that docs not require of the presence of a lattice, as the one explained above. However, dopants such as Arsenic that can migrate by coupling with a vacancy require of the details of a lattice Monte Carlo model $[34,35]$, where the simulation includes all the different lattice positions. 
As mentioned earlier, most of the ab initio simulations existing in the literature are zero temperature calculations, providing the migration energies of the dopants but not the actual rates for migration. In our simulations, in order to obtain the probabilities for Boron migration we fitted the jump rate pre-factor to experimental values of Boron diffusivity in Silicon [37]. In a kinetic Monte Carlo box of $80 \times 80 \times 80 \mathrm{~nm}^{3}$, with periodic boundary conditions, we included one Boron substitutional and one silicon self-interstitial, using an ad-hoc value for the pre-factor for the boron interstitial diffusivity. The final boron diffusivity was obtained from the total number of hops after adjusting for size effects and interstitial concentration. The initial value of the pre-factor was modified until the calculated diffusivity reproduced the experimental results for different temperatures.

A step further in the complexity of a Monte Carlo simulation of defect diffusion is the possibility of clustering of dopants with silicon defects. Recent LDA calculations have provided of a large data base of binding energies and reactions of Boron atoms and Silicon self-interstitials. The numerous possible combinations for dopant and defect clusters make this a very complicated calculation. However, the combined effort of Monte Carlo simulations, that can explore the possibility of different reaction paths together with accurate and detailed LDA calculations allowed for the production of these large set of data [30].

All these reaction rates are fundamental parameters for any Monte Carlo simulation of defect and dopant diffusion in Silicon. Only the initial conditions will change depending on the type of experiment that needs to be simulated, such as ion implantation, oxidation enhanced diffusion (OED), etc. In particular, for the simulation of ion implantation it is necessary to obtain the initial configuration of the vacancies and interstitials created by the energetic ions bombarding the sample. This defect distribution can be obtained from a 
binary collision model, such as UT-Marlowe [37], or from a molecular dynamics simulation of the damage produced by an energetic ion $[28,38]$. The former is computationally more efficient, improving the statistics of the simulation. However, it is not always valid. For the case of light ion bombardment, such as Boron implantation, simple models such as binary collision simulations, give an accurate description of the damage produced by the ion, since mostly isolated pairs of vacancies and interstitials (Frenkel pairs) will be formed during the collision cascade. For the case of heavier ions, such as Arsenic, molecular dynamics has shown that the collision of a single ion can produce an amorphous zone, that can not be accurately described by binary collision models, since these highly disordered regions are the result of multiple close collisions [38]. As a result of this study it was observed that the total fraction of defects produced by heavy ions is twice the value obtained from binary collision models. The stability of these amorphous structures was also studied by $\mathrm{MD}$ for annealing at different temperatures. It was observed that these structures anneal at low temperatures in just a few picoseconds, but the stability of the amorphous pockets increases with the initial size of the disordered zone, and therefore, with the ion mass and energy. All these factors must be taken into account when modeling the implantation and diffusion of defects in Silicon if a realistic model wants to be constructed.

In a Monte Carlo simulation of ion implantation, the time between the arrival of two consecutive cascades is given by the dose rate. During these time defects are allowed to diffuse, therefore, dose rate effects are directly included in these simulations. As an illustration, in figure 1 we show a snap-shot of a simulation of $5 \mathrm{keV}$ Boron implantation. Only a small slice of the total simulation box is shown here, for two different times: figure 1(a) for time $=1.4 \mathrm{~s}$ corresponding to a dose of $2 \times 10^{12}$ ions $/ \mathrm{cm}^{2}$ and figure $1(\mathrm{~b})$ for time $=$ $19 \mathrm{~s}$ corresponding to a dose an order of magnitude higher, $2 \times 10^{13}$ ions $/ \mathrm{cm}^{2}$. White circles represent vacancies and gray circles are interstitials. Larger circles represent clusters of 
vacancies or interstitials. Black dots corresponds to Boron atoms and Boron complexes. Observe that during the implantation most of the vacancies are already in clusters, while interstitials are mostly single. In this simulations, dopant ions are set in interstitial sites after the collision cascade. Periodic boundary conditions are used for $\mathrm{y}$ and $\mathrm{z}$ directions while the front and back surfaces act as perfect sinks.

\section{Applications}

One of the major efforts in kinetic Monte Carlo simulations has been the study of transient enhanced diffusion (TED) of Boron in Silicon. L. Pelaz et al [39] developed a model for Boron migration and clustering that successfully reproduces transient enhanced diffusion occurring in MBE grown Boron layers, implanted with $\mathrm{Si} 40 \mathrm{keV}$ at a dose of $9 \times 10^{13}$ ions $/ \mathrm{cm}^{2}$ and annealed at $800^{\circ} \mathrm{C}$. Values obtained from molecular dynamics for the migration energy of silicon defects [15] and from ab initio calculations for the migration energy of Boron [3] were used in these simulations. The energetics and reactions for Boron clustering were obtained by fitting to the experimental profiles. From these simulations it was concluded that a Boron precursor, such as BI2 (one Boron atom a two silicon self interstitials) was necessary in order to reproduce the different annealed profiles, in particular to obtain a fixed peak, as the one observed in the experimental data. This Boron cluster is formed in the initial stages of the cascade damage or the high temperature annealing, when the Si interstitial super-saturation is very high. According to this study, these defects act as nucleation centers for the formation of B-rich clusters during annealing, responsible for the electrically inactive component of the Boron concentration profile. The experimental time dependence of TED was accurately reproduced with this model for simulations at $800 \mathrm{C}$. 
We have developed a similar model for transient enhanced diffusion of Boron. In our case, the different binding energies and possible B-I clusters were calculated using ab initio simulations, as explained in the section above. We centered the study on the simulation of the transient enhanced diffusion of as implanted Boron profiles under different annealing temperatures and times, and different energies of the implanted ion. Figure 2 shows the Boron concentration depth profile for a $40 \mathrm{keV}$ Boron implant at $2 \times 10^{14}$ ions $/ \mathrm{cm}^{2}$ after annealing at $700^{\circ} \mathrm{C}$ for 240 minutes (figure $2(\mathrm{a})$ ) and annealing at $900^{\circ} \mathrm{C}$ for 5 minutes (Figure 2(b)). The kinetic Monte Carlo results for the total Boron concentration profiles (circles) are compared with the SIMS measurements for these conditions (lines), and a good agreement is observed for all different temperatures and annealing times studied [30].

In order to understand how Boron diffusion and clustering occurs we study the evolution of the different B-I clusters as a function of time during high temperature annealing. In figure 3 we show the concentration of the most important B-I clusters as a function of time during a $800^{\circ} \mathrm{C}$ anneal, for a $\mathrm{B} 40 \mathrm{keV}, 2 \times 10^{14} \mathrm{~cm}^{-2}$ implantation. In this plot we also include the percentage of active Boron as a function of time (solid line, right ordinate). After the implantation most of the B-I clusters are in the form of BI (one Boron atom and one Silicon self-interstitial, open squares in figure 3) and BI2 (filled circles). Only $56 \%$ of the Boron is active after implantation. At this time, all vacancies are in clusters and most of the interstitials are single defects. During the early stages of the high temperature anneal interstitials agglomerate to form clusters and vacancy clusters grow and dissociate due to their lower binding energies (see section II). This can be observed in figure 4, where we show the average cluster size of vacancies and interstitials as a function of annealing time (left ordinate). The high concentration of vacancies makes the recombination with B-I complexes very likely and therefore a reduction in the concentration of these clusters is observed during the initial annealing stages, while the concentration of Boron substitutional increases, with a total activation of $98 \%$ at $10^{-2}$ seconds. After approximately 10 seconds all 
the vacancies have recombined with interstitials, dopants or the surface and no more vacancies are left in the bulk (see figure 4). It is after the release of all the vacancies from the system when the interstitial clusters start to grow to later release single interstitials, as observed in earlier simulations [29].

Also in figure 4 we show the magnitude of boron TED, the total diffusion length (right ordinate, solid line). The boron diffusion length increases when all vacancies have disappeared from the system. It is interesting to note that only $4 \mathrm{~nm}$ of the total boron diffusion length of $48 \mathrm{~nm}$ occurs during the final interstitial cluster dissolution. Eight $\mathrm{nm}$ occur during the first $10 \mathrm{~s}$ and $36 \mathrm{~nm}$ during interstitial cluster growth, that is, before dissolution of the larger clusters. It is during the growth and dissociation of the interstitial clusters when Boron atoms can migrate and clustering of Boron atoms and silicon-self interstitials occurs. This can be observed in figure 3, where the concentration of B-I clusters of the type B3I (squares) starts to increase after 10s of anneal. The formation of these B3I clusters implies a reduction in the total boron active. When the transient is over only $72 \%$ of the total Boron is active. It is interesting to note the existence of a time gap, between $10^{-2}$ and $10 \mathrm{~s}$ when $98 \%$ of all the Boron is active.

From the simulations described above we have extracted the Boron diffusivity as a function of annealing temperature. This has been obtained by counting the total number of hops of the Boron interstitials in the simulation until no more self-interstitials are left in the bulk and all the Boron atoms are either in substitutional sites or in stable cluster, such as B3I. In figure 5 we present the results for the Boron diffusivity for three different temperatures, $700^{\circ} \mathrm{C}, 800^{\circ} \mathrm{C}$ and $900^{\circ} \mathrm{C}$ (left ordinate, circles). The dependence with temperature obtained from this plot for the Boron diffusivity is: $\mathrm{D}_{\mathrm{B}}=2.2 \times 10^{-2} * \exp (-2.7 \mathrm{eV} / \mathrm{KT}) \mathrm{cm}^{2} / \mathrm{s}$, in good agreement with the result of Solmi and Baruffaldi, obtained from different experimental observations [40] of $D_{B}=2.2 \times 10^{-2} * \exp (-2.5 \mathrm{eV} / \mathrm{KT}) \mathrm{cm}^{2} / \mathrm{s}$. Also in figure 4 (right ordinate, 
squares) we show the total diffusion length at different temperatures, in nm. As expected, and previously reported in some experimental observations [41], the total diffusion length is lower for higher annealing temperatures at the end of the transient. Our model, is therefore, able to predict the temperature dependence of the transient enhanced diffusion. The results of the simulations were also compared with SIMS profiles for these three temperatures with good agreement for different annealing times.

The energy dependence of TED has also been studied using k-MC simulation and compared with experimental results. In figure 6 we present the results for Boron concentration depth profiles at the same dose reported above, $2 \times 10^{14}$ ions $/ \mathrm{cm}^{2}$, and two different energies (a) $20 \mathrm{keV}$ and (b) $80 \mathrm{keV}$, both the as-implanted profiles (straight line are the SIMS measurements, circles are the k-MC), and the profiles after annealing at $800^{\circ} \mathrm{C}$ for 60 minutes (dashed lines are SIMS measurements, squares are simulations). Observe again the good agreement between experiments and our simulation model.

Similarly to the case above for the temperature dependence of TED we have calculated the Boron diffusivity as a function of energy. In figure 7 we show the results for the Boron diffusivity (left ordinate, circles) as a function of energy for three different energies $(20,40$ and $80 \mathrm{keV})$, and the same dose $\left(2 \times 10^{14} \mathrm{ions} / \mathrm{cm}^{2}\right)$ and annealing temperature $\left(800^{\circ} \mathrm{C}\right.$ for 60 minutes). As observed experimentally [42] there is a very small dependence of TED with energy. This dependence with energy can be related to the location of the boron profile with respect to the surface, and consequently to the total number of interstitials recombined at the surface, or the total number of interstitials remaining in the bulk that are able to induce Boron migration. In figure 7 we present the total number of interstitials recombined at the surface after the 60 minutes anneal at $800^{\circ} \mathrm{C}$ for the three different energies (left ordinate, squares). This number increases as the energy increases since the maximum of the damage 
concentration profile is deeper in the bulk. The difference in the total number of interstitials available for TED is responsible for this energy dependence.

The versatility of a kinetic Monte Carlo simulation allows for studies of different experimental conditions. Recently, the influence of Carbon and Boron in the experimental measurements of Silicon self-interstitial diffusivity has been explored by simulating the enhancement due to oxidation [43]. From this study it was found that modest levels of carbon $\left(10^{17} \mathrm{~cm}^{-3}\right)$ significantly reduce the effective interstitial diffusivity. A fixed interstitial population at the surface was considered in these simulations and the interstitial depth profiles were monitored with time. From fitting these profiles to a complementary error function the silicon self-interstitial diffusivity was obtained. The same procedure was followed for different carbon concentrations and temperatures. In figure 8 we show the values of the effective diffusivities as obtained from these simulations (solid lines). Notice in this figure that carbon not only changes the prefactor for diffusivity, but also dramatically increases the effective self-interstitial migration energy from $0.9 \mathrm{eV}$ for no carbon to $1.98 \mathrm{eV}$ for a carbon concentration of $10^{18} \mathrm{~cm}^{-3}$. These results suggest an explanation for the vast spread in the experimentally determined interstitial diffusivity. In the same figure the results of the simulation are compared to two general classes of experiments. One class involves monitoring the diffusion of metal tracers and follows the general trend of shallow slopes at the higher values of diffusivity and steeper slopes for lower values of the diffusivity [44-56] - with a few notable exceptions [50-53], (see ref. [44] for a detailed comparison of the experiments). The second class of experiments monitors the spreading of doped marker layers during oxidation enhanced diffusion (OED). In this case, a much lower diffusivity has been measured and activation energies for migration were determined to be in the range of $3.1 \mathrm{eV}[3,5,54,55]$. These results cannot be explained by carbon trapping. 
Simulation of the spreading of boron spikes during OED has shown that Boron acts as an effective trap for interstitials, due to the formation of both mobile and immobile complexes. A kinetic Monte Carlo simulation was performed under the same conditions as the experiments by Gossmann et al [56]. For these simulations the matrix consists of a set of boron delta-doped spikes with a background carbon concentration of $5 \times 10^{17} \mathrm{~cm}^{-3}$. The surface concentration of silicon self-interstitials was maintained at $2 \times 10^{10} \mathrm{~cm}^{-3}$ and the annealing temperature was $810^{\circ} \mathrm{C}$. In figure 9 we show the results of the simulation for a Boron concentration of $10^{18} \mathrm{~cm}^{-3}$. Observe the excellent agreement with the experimental data.

The effective interstitial diffusivity was extracted from this simulation by two methods. One method consists of extracting the width of the as-deposited and annealed spikes to calculate the diffusivities. In this method, the diffusivity of the self interstitial is extracted from the B diffusivity profiles by fitting the spreading of the peaks with a Gaussian, assuming the B diffusivity is proportional to the self-interstitial concentration. This method gives similar results to Gossmann's analysis. In this case, clusters of boron and interstitials are a small fraction of the total boron concentration, so the spreading of the peaks is expected to give a good measure of the effective diffusivity. The self-interstitial concentration profiles were also analyzed. From this analysis it was found that the spike spreading method gives a diffusivity of $2.2 \times 10^{-12} \mathrm{~cm}^{2} / \mathrm{s}$ while a fit to the interstitial concentration profile yields an effective diffusivity of $3.6 \times 10^{-12} \mathrm{~cm}^{2} / \mathrm{s}$. The difference is within the accuracy of the simulation and the result supports the idea that spike width spreading measures the interstitial profile. However, it is interesting to note that both of these methods give a diffusivity which is orders of magnitude smaller that the diffusivity measured in a simulation with no boron, only carbon, as the results in figure 8 indicate. Yet Boron comprises only $\sim 25 \%$ of the total number of traps within the delta-doped region. The cause for the precipitous drop in effective diffusivity due to boron is a results of the details of the 
interactions between boron and self-interstitials, and between carbon and self-interstitials. In the case of carbon, the CI complex (i.e. the first order trap) is mobile and has a diffusivity of $\sim 2 \times 10^{-9} \mathrm{~cm}^{-3}$ and $810^{\circ} \mathrm{C}$, whereas in the case of boron the lowest energy state of the simple boron-interstitial complex is immobile [3]. Though the boron-interstitial complex can "kick out" a mobile interstitial boron atom, the energy difference between these two estates in about $0.4 \mathrm{eV}$ [3] which implies that the mobile interstitial boron concentration will be less than $2 \%$ of the concentration of the immobile boron-interstitial pairs at $810^{\circ} \mathrm{C}$. Threfore, the B-I interaction will yield an effective diffusivity for the self interstitial comparable to that expected for fixed traps [56]. The carbon and boron trapping can reconcile the large difference in diffusivity obtained from the metal diffusion experiments and B marker layer experiments [44-56].

From the examples described above we can conclude that kinetic Monte Carlo simulations can be used as predictive models for phenomena such as transient enhanced diffusion of Boron, or self-interstitial trapping by carbon atoms, as long as there is a proper description of all the fundamental processes occurring in the matrix. However, these models are still too computationally intensive to be used by the industry on a regular basis. Nevertheless, they can provide the most important reaction paths, and a reduced model can be constructed. This model can then be implemented into continuum descriptions such as ALAMODE or SUPREME. In fact, this type of simulations were performed using ALAMODE with the parameters extracted from ab initio and molecular dynamics. In figure 10 we show the results from the simulation of $\mathrm{B} 40 \mathrm{keV}, 2 \times 10^{14} \mathrm{ions} / \mathrm{cm}^{2}$ and annealing at $800^{\circ} \mathrm{C}$ as obtained from Monte Carlo (symbols), ALAMODE (dashed line) and SIMS profiles (continuum line), with good agreement between all three. These simulation models can be very helpful to the semiconductor industry in the development of future devices.

\section{Conclusions}


We have described in this paper some of the most recent results of defect and dopant diffusion using kinetic Monte Carlo simulations. Good agreement is found between experiments and simulations for those cases explained here, that is, medium implant energies (5-50 keV) and low doses $\left(\sim 10^{14}\right.$ ions $\left./ \mathrm{cm}^{2}\right)$. However, several issues still remain unknown regarding simulations of those experimental conditions considered in the industry for future devices, that is, ultra-low energies (below $1 \mathrm{keV}$ ) and high doses $\left(>10^{15}\right.$ ions $/ \mathrm{cm}^{2}$ ). Parameters such as recombination efficiency of defects and dopants at surfaces are critical when the implantation energies are reduced, and most of the damage is close to the surface, as well as for high dopant concentrations near the surface. The interactions of defects with the silicon-oxide/silicon interface needs to be understood and implemented in these Monte Carlo codes, in order to describe systems such as those considered for shallow junction formation. Moreover, Fermi level effects on the interstitial concentration and dopant diffusion need to be taken into account when the dopant concentration is high and we have an extrinsic semiconductor even at the high temperatures of the anneal. All these effects can be included in the Monte Carlo model regarding the different reaction rates and paths are known. Therefore, further basic simulations, molecular dynamics and ab initio, are necessary to understand the physics of interfaces and clustering at high concentrations. Further development of these type of simulations can include the description of interactions between defects and the dislocations present in the material. 


\section{References}

[1] C. S. Nichols, C. G. Van de Walle, S. T. Panmtelides, Phys. Rev. B40, 5458 (1989)

[2] W.A. Harrison Mat. Res. Soc. Symp. Proc. in Defects and Diffusion in Silicon Processing, Vol. 469, p. 211, Ed. T. Diaz de la Rubia, S. Coffa, P. A. Stolk, C. S. Rafferty, (1997)

[3] J. Zhu, T. Diaz de la Rubia, L. H. Yang, C. Mailhiot, G. H. Gilmer, Phys. Rev. B54, 4741 (1996)

[4] J. Nelson, private communication

[5] G. D. Watkins, Mat. Res. Soc. Symp. Proc. in Defects and Diffusion in Silicon Processing, Vol. 469, p. 139, Ed. T. Diaz de la Rubia, S. Coffa, P. A. Stolk, C. S. Rafferty, (1997)

[6] P.A. Stolk, H. J. Gossmann, D.J. Eaglesham, D.C. Jacobson, J.M. Poate, Appl. Phys. Lett. 66, 568 (1995)

[7] P. J. Bedrossian, M.-J. Caturla, T. Diaz de la Rubia, Appl. Phys. Lett. 70, 176 (1997)

[8] S. Prussin, K. S. Jones, J. of Electro. Soc. 137, 1912 (1990)

[9] P. Ehrnhart, H. Zillgen, Mat. Res. Soc. Symp. Proc. in Defects and Diffusion in Silicon Processing, Vol. 469, p. 175, Ed. T. Diaz de la Rubia, S. Coffa, P. A. Stolk, C. S. Rafferty, (1997)

[10] T. Diaz de la Rubia, G. H. Gilmer, Phys. Rev. Lett. 74, 2507 (1995)

[11] M. J. Caturla, T. Diaz de la Rubia, G. H. Gilmer, Nucl. Instrum. Meth. B106, 1, (1995)

[12] R. Smith, K. Beardmore, A. Gras-Marti, R. Kirchner, Nucl. Instrum. Meth. B102, $211(1995)$

[13] J. M. Perlado, Jour. Nucl. Mat. 251, 98 (1997), Jour. Nucl. Mat. 233, 1096 (1996) 
[14] P. B. Rasband, A. P. Horsfield, P. Clancy, Phil. Mag. B 73, 71 (1996)

[15] G. H. Gilmer, T. Diaz de la Rubia, D. M. Stock and M. Jaraiz, Nucl. Instrum. and Methods B102, 247 (1995)

[16] M. Tang, L. Colombo, J. Zhu, T. Diaz de la Rubia, Phys. Rev. B55, (1997)

[17] N. Cuewndet, T. Halicioglu, w. A. Tiller, Appl. Phys. Lett. 69, 4071 (1996)

[18] S. Libertino, S. Coffa, G. Franzo, F. Priolo, J. Appl. Phys. 78, 3867 (1995)

[19] P. J. Partyka, R. S. Averback, K. Norlund, I. K. Robinson, P. Ehrhart, T. Diaz de la Rubia, M. Tang, Mat. Res. Soc. Symp. Proc. in Microstructure Evolution during irradiation, Vol. 439, p. 175, Ed. I. M. Robertson, G. S. Was, L. W. Hobbs, T. Diaz de la Rubia (1997)

[20] K. Norlund, P. Partyka, R. S. Averback, Mat. Res. Soc. Symp. Proc. in Defects and Diffusion in Silicon Processing, Vol. 469, p. 199, Ed. T. Diaz de la Rubia, S. Coffa, P. A. Stolk, C. S. Rafferty, (1997)

[21] U. Gösele, A. Plö $\beta 1$ and T. Y. Tan in Process Physics and Modeling in Semiconductors Technology, Ed., by G. R. Srinivassan, C. S. Murthy and S. T. Dunham (Electrochemical Society, Pennington, New Jersey, 1996) p. 309

[22] M. D. Giles, Yu Shaofeng, H. W. Kennel, P. A. Packan, Mat. Res. Soc. Symp. Proc. in Defects and Diffusion in Silicon Processing, Vol. 469, p. 253, Ed. T. Diaz de la Rubia, S. Coffa, P. A. Stolk, C. S. Rafferty, (1997)

[23] M. E. Law, K. S. Jones, S. K. Earles, A. D. Lilak, J-W Xu, Mat. Res. Soc. Symp. Proc. in Microstructure Evolution during irradiation, Vol. 439, p. 3, Ed. I. M. Robertson, G. S. Was, L. W. Hobbs, T. Diaz de la Rubia (1997)

[24] C. S. Rafferty, G. H. Gilmer, M. Jaraiz, D. Eaglesham, H.-J. Gossmann, Appl. Phys. Lett. 68, 2395 (1996)

[25] P. B. Griffin, J. D. Plummer, in Process Physics and Modeling in Semiconductors Technology, Ed., by G. R. Srinivassan, C. S. Murthy and S. T. Dunham (Electrochemical Society, Pennington, New Yersey, 1996) p. 101 
[26] J. D. Plummer, P. B. Griffin, Nucl. Instrum. and Methods B102, 160 (1995)

[27] A. D. Lilak, S. K. Earles, K. S. Jones, M. E. Law, International electron devices meeting, IEDM Technical Digest Washington, D.C., IEEE p. 493 (1997)

[28] H. L. Heinisch, Nucl. Instrum. and Methods B102, 47 (1995)

[29] M. Jaraiz, G. H. Gilmer, J. M. Poate, Appl. Phys. Lett. 68, 409 (1996)

[30] M. J. Caturla, A. Lilak, M. D. Johnson, J. Zhu, M. Giles, T. Diaz de la Rubia, M. Law, (to be submitted to J. Appl. Phys.)

[31] H. Bracht, N. A. Stolwijk, H. Mehrer, Proc. Electochem. Soc. 94-10, 593 (1994)

[32] L. Colombo, private communication

[33] J. Zhu, Mat. Res. Soc. Symp. Proc. in Defects and Diffusion in Silicon Processing , Vol. 469, p. 151, Ed. T. Diaz de la Rubia, S. Coffa, P. A. Stolk, C. S. Rafferty, (1997)

[34] O. Pankratov, H. Huang, T. Diaz de la Rubia, C. Mailhiot, Phys. Rev. B (1997)

[35] S. T. dunham, C. D. Wu, J. Appl. Phys. 78, 2362 (1995)

[36]A. D. Kurtz, R. Yee, J. Appl. Phys. 31, 303 (1960)

[37] S. Tian, S. J. Morris, B. Obradovic, M. f. Morris, G. Wang, G. Balamurugan, A. F. Tasch, C. Snell, UT-Marlowe, Version 4.0 (1996)

[38] M.-J. Caturla, L. A. Marques, T. Diaz de la Rubia, G. H. Gilmer, Phys. Rev. B54, 16683 (1996)

[39] L. Pelaz, M. Jaraiz, G. H. Gilmer, H. J. Gossmann, C. S. Rafferty, D. J. Eaglesham, J. M. Poate, Appl. Phys. Lett. 70, 2285 (1997)

[40] S. Solmi and F. Baruffaldi, J. Appl. Phys. 69, 2135 (1991)

[41]P. a. Packan, J. D. Plummer, Appl. Phys. Lett. 56, 1787 (1990)

[42] H. S. Chao, P. B. Griffin, J. D. Plummer, C. S. Rafferty, Appl. Phys. Lett. 69, 2113 (1996)

[43] M. D. Johnson, M.-J. Caturla, T. Diaz de la Rubia, J. Appl. Phys. (in press)

[44] H. Bracht, N. A. Stolwijk and H. Mehrer, Proc. Electrochem. Soc. 94-10, 593 (1994) 
[45] H.-J. Gossmann, C. S. Rafferty, H. S. Luftman, F. C. Uterwald, T. Boone and J. M. Poate, Appl. Phys. Lett. 63, 639 (1993)

[46] F.F. Morehead, in Defects in Electronic Materials, edited by M. Stabola, S. J. Pearton, and G. Davies (Materials Research Society, Pittsburgh, 1988), p.99

[47] H. Zimmermann and H. Ryussel, Appl. Phys. A 55, 131 (1992)

[48] W. Wijaranakula, J. Appl. Phys, 67, 7624 (1990)

[49] G. B. Bronner and J. D. Plummer, J. Appl. Phys, 61, 5286 (1987)

[50] T. Y. Tan and U. Gossele, Appl. Phys. A 37, 1 (1985)

[51] C. Boit, F. Lau, and R. Soittig, Appl. Phys. A 50, 197 (1990)

[52] K. Taniguchi, D. A. Antoniadis and Y. Matsiushita, Appl. Phys. Lett. 42, 961 (1983)

[53] K. Ghaderi, G. Hobler, M. Budil, L. Mader and H. J. Schulze, J. Appl. Phys. 77, $1320(1995)$

[54] H.-J. Gossman, G. H. Gilmer, C. S. Rafferty, F. C. Unterwald, T. Boone, J. M. Poate, H. S. Luftman, and W. Frank, J. Appl. Phys. 77, 1948 (1995)

[55]. A. M. Agarwal and S. T. Dunham., Appl. Phys. Lett. 63, 800 (1993)

[56] H.-J. Gossmann set al., J. Appl. Phys. 77, 1948 (1995)

[57] P. M. Fahey, P. B. Griffin, J. D. Plummer, Rev. Mod. Phys. 61, 289 (1989) 


\section{Figure captions}

Figure 1. Snap-shot of the defect distribution in a Monte Carlo simulation. We only show a box of $10 \times 10 \times 10 \mathrm{~nm}^{2}$, for two different doses: (a) $2 \times 10^{12}$ ions $/ \mathrm{cm}^{2}$ and (b) $2 \times 10^{13}$ ions $/ \mathrm{cm}^{2}$. White circles represent vacancies and greys are interstitials. The black circles are Boron atoms or Boron complexes. Larger circles represent clusters.

Figure 2. Depth concentration profiles for B $40 \mathrm{keV}$ implantation at a dose of $2 \times 10^{14}$ ions $/ \mathrm{cm}^{2}$ after annealing at (a) $700^{\circ} \mathrm{C}$ for 240 minutes and (b) $900^{\circ} \mathrm{C}$ for 5 minutes. The circles are the results from Monte Carlo simulation of the total Boron concentration profile. The solid line is the SIMS measurement. The squares are the Monte Carlo values for the Boron concentration in Boron clusters.

Figure 3. Time history of the substitutional and clustered boron fractions following a $800^{\circ} \mathrm{C}$ anneal of the $40 \mathrm{keV}$ boron profile shown in Fig. 1. The percentage of Boron active is indicated in the right ordinate, while the concentration of B-I complexes are in the left ordinate. The boron substitutional fraction increases from $56 \%$ to $98 \%$ after $10^{-2} \mathrm{~s}$ but decreases again to approximately $71 \%$ after the vacancies disappear, and interstitial and B3I clusters start to grow.

Figure 4. Average vacancy and Interstitial cluster size versus time during a $800^{\circ} \mathrm{C}$ anneal of $40 \mathrm{keV}$ boron implanted silicon. Also shown on the right ordinate is the total boron diffusion length as a function of time (-).

Figure 5. Boron diffusivity as a function of annealing temperature as extracted from Monte Carlo simulations (circles, left ordinate). Also in the right ordinate and with squares we show the total diffusion length for these temperatures, and for the case of $40 \mathrm{keV} \mathrm{B}$ at 
$2 \times 10^{14}$ ions $/ \mathrm{cm}^{2}$. Observe that the total diffusion length increases with decreasing temperature, in agreement with the experiments.

Figure 6. Depth concentration profiles for B implantation at a dose of $2 \times 10^{14}$ ions $/ \mathrm{cm}^{2}$ after annealing at $800^{\circ} \mathrm{C}$ for 60 minutes for two different implant energies (a) $20 \mathrm{keV}$ and (b) $80 \mathrm{keV}$. The circles are the results from Monte Carlo simulation of the total Boron concentration profile. The solid line is the SIMS measurement. The squares are the Monte Carlo values for the Boron concentration in Boron clusters.

Figure 7. Boron diffusivity as a function of implant energy as extracted from Monte Carlo simulations (circles, left ordinate). Also in the right ordinate and with squares we show the number of Silicon self-interstitials recombined at the surface as a function of energy. Observe that as the energy decreases the number of available interstitials decreases, therefore, the transient enhanced diffusion decreases slightly with energy.

Figure 8. Comparison between experimentally measured self-interstitial diffusivity (dashed lines and unfilled symbols) and simulated effective diffusivities (solid lines and filled symbols). The experimental results 1-13 correspond to references [40-52] respectively.

Figure 9. As deposited (thin dashed) and annealed (solid) Boron concentration profiles from Gossmann et al. [56]. The as-deposited profile is used as the starting point for the kinetic Monte Carlo simulations. The simulated profiles (diamonds) were obtained after annealing at $810^{\circ} \mathrm{C}$ for $15 \mathrm{~min}$, with a surface concentration maintained at $2 \times 10^{10} \mathrm{~cm}^{-3}$.

Figure 10. Depth concentration profile for Boron $40 \mathrm{keV}$ implant, $2 \times 10^{14}$ ions $/ \mathrm{cm}^{2}$, annealed at $800^{\circ} \mathrm{C}$ for 60 minutes as obtained from Monte Carlo simulations (symbols), 
SIMS measurements (dashed line) and continuum simulations (solid lines). Observe the good agreement between the simulations that use parameters obtained from $\mathrm{MD}$ and $\mathrm{ab}$ initio, and the experimental profiles. 
FIGURE 1(a)

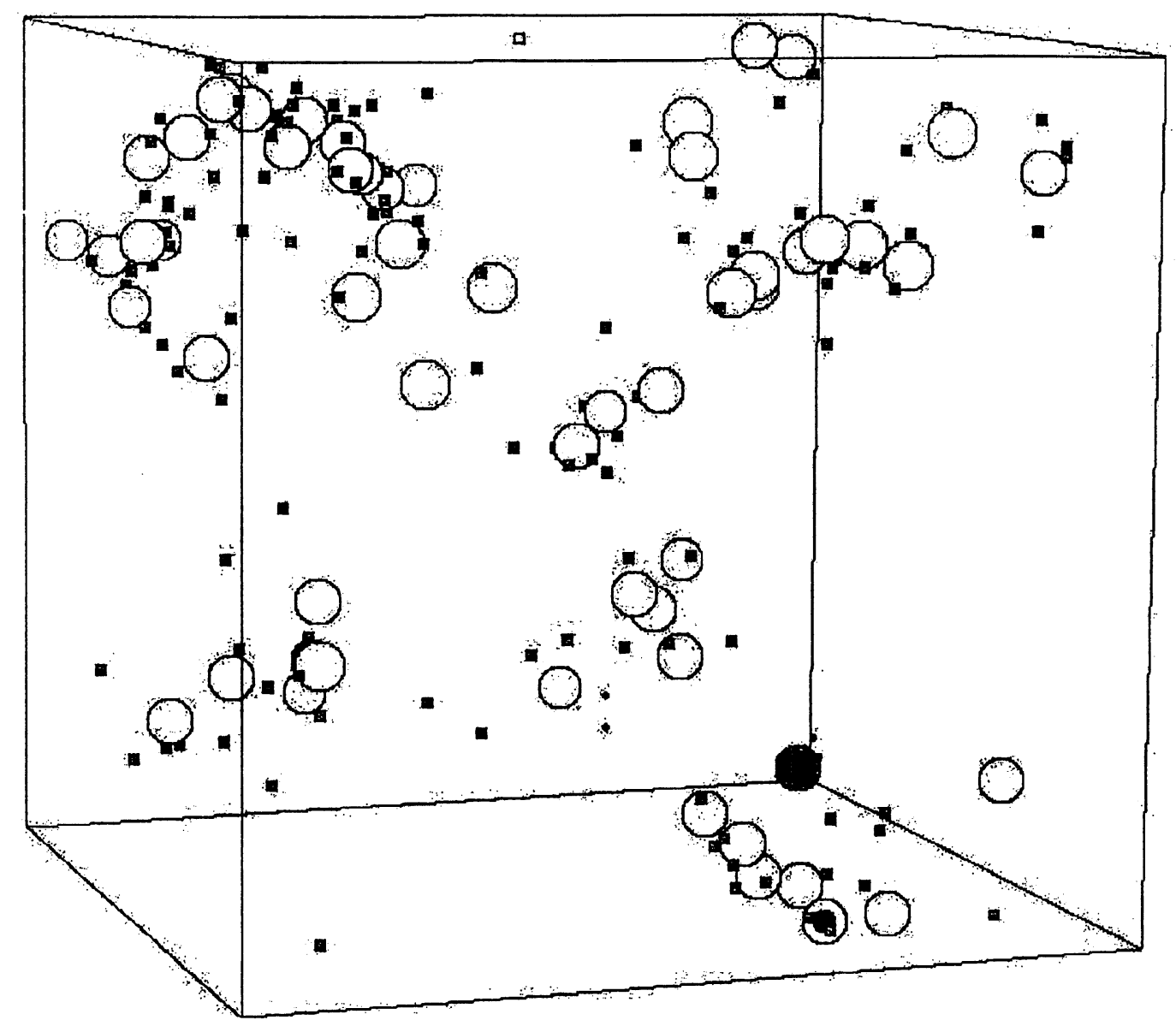




\section{FIGURE 1(b)}

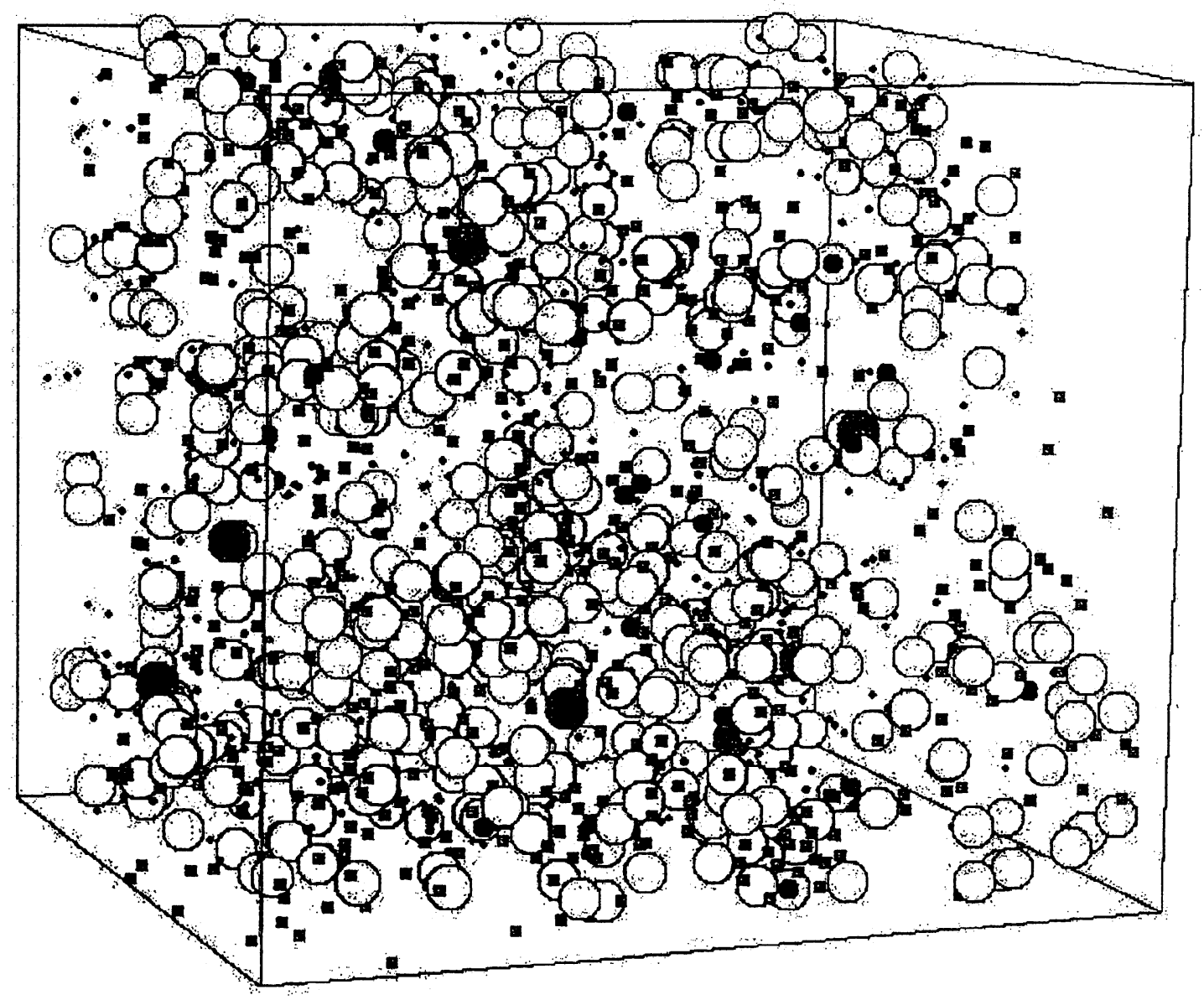


FIGURE 2(a)

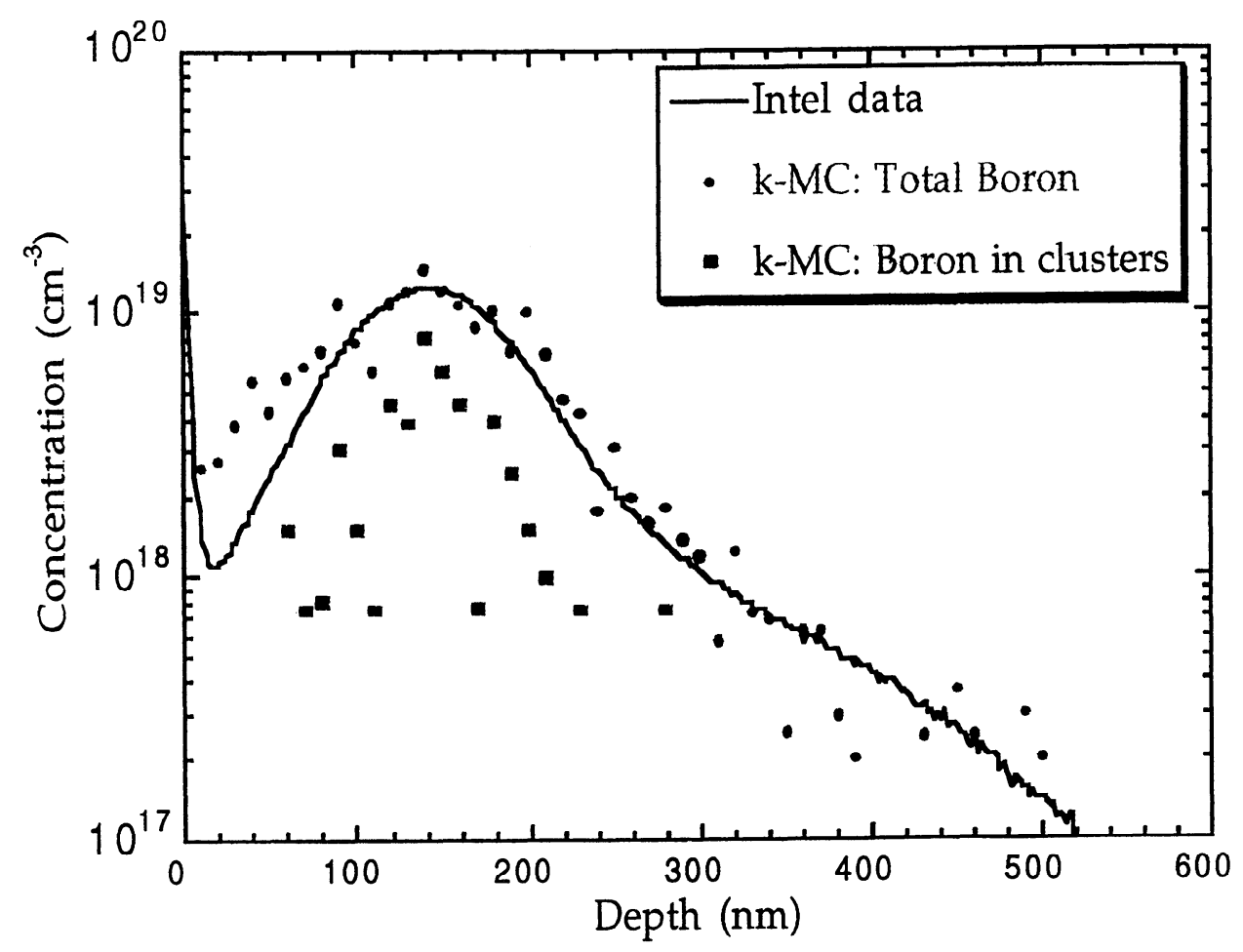


FIGURE 2(b)

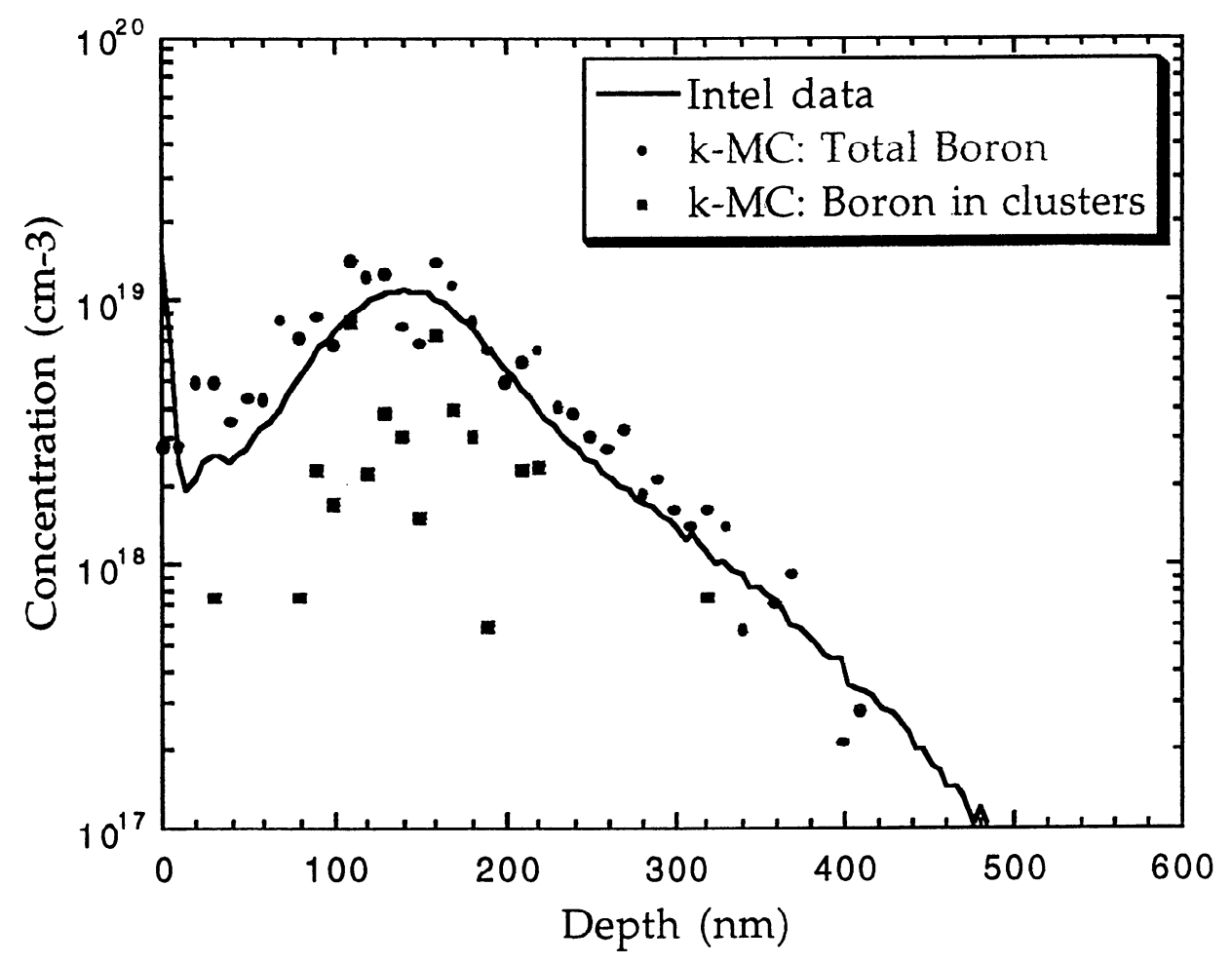


FIGURE 3

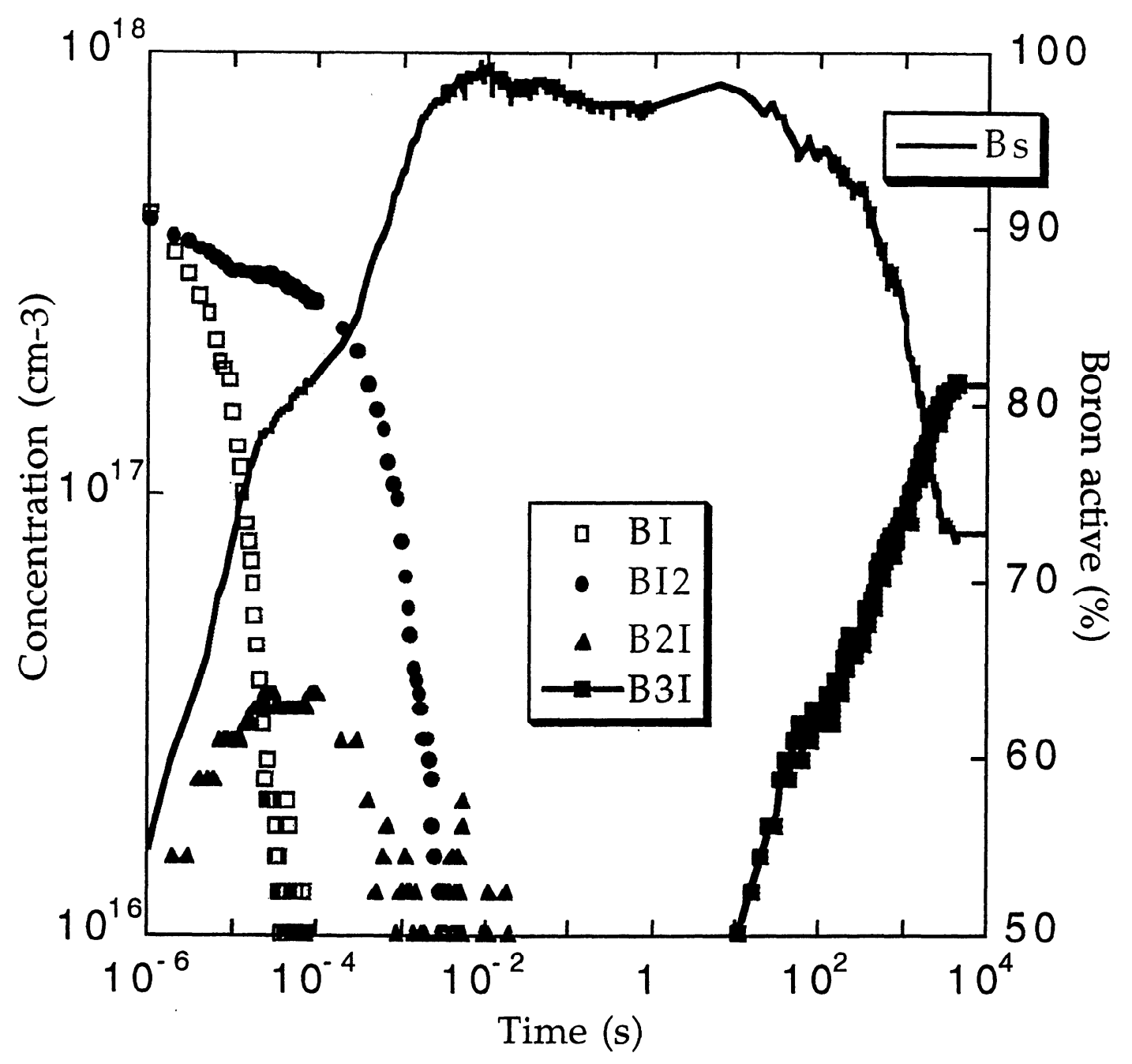


FIGURE 4

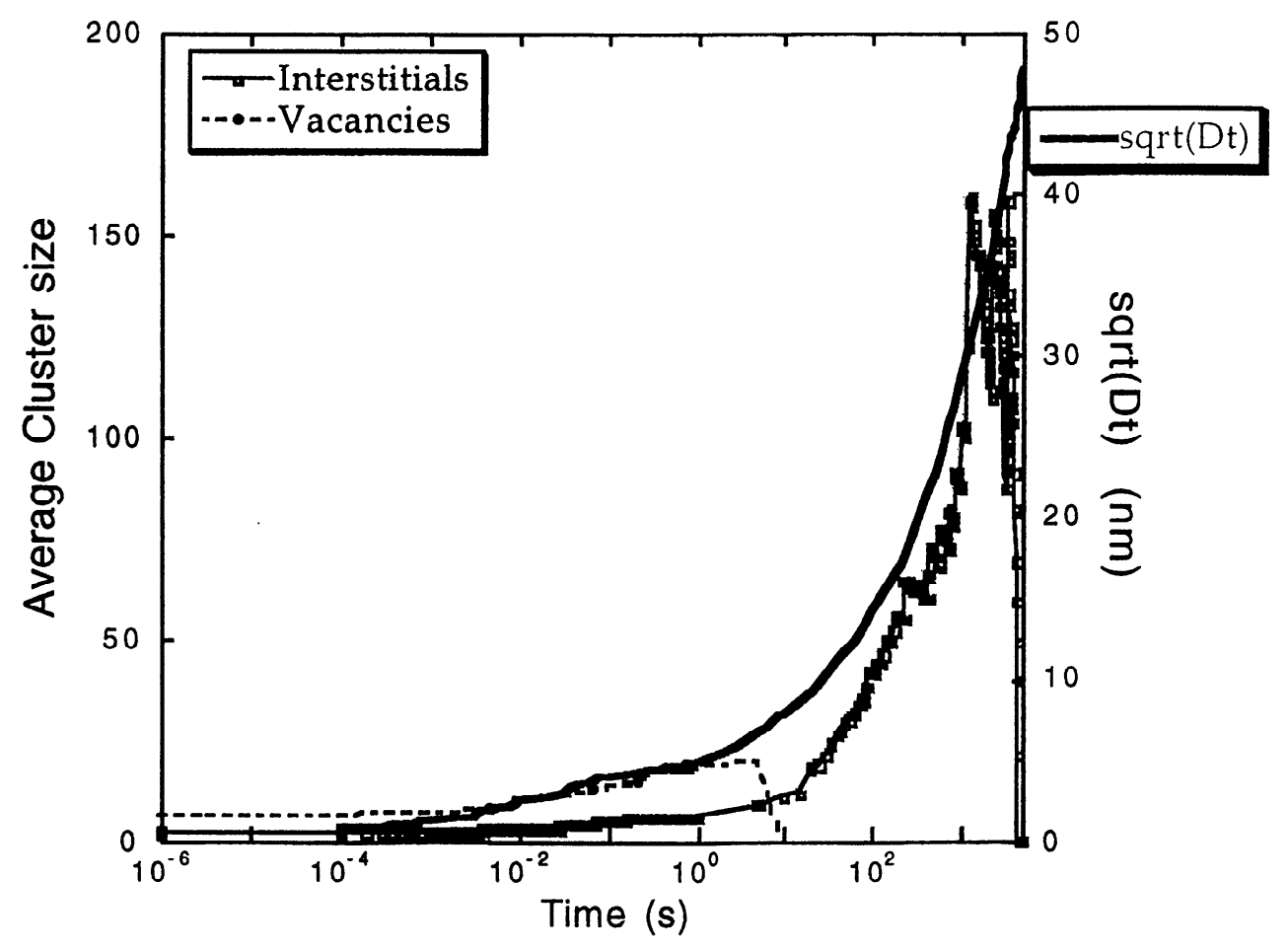




\section{FIGURE 5}

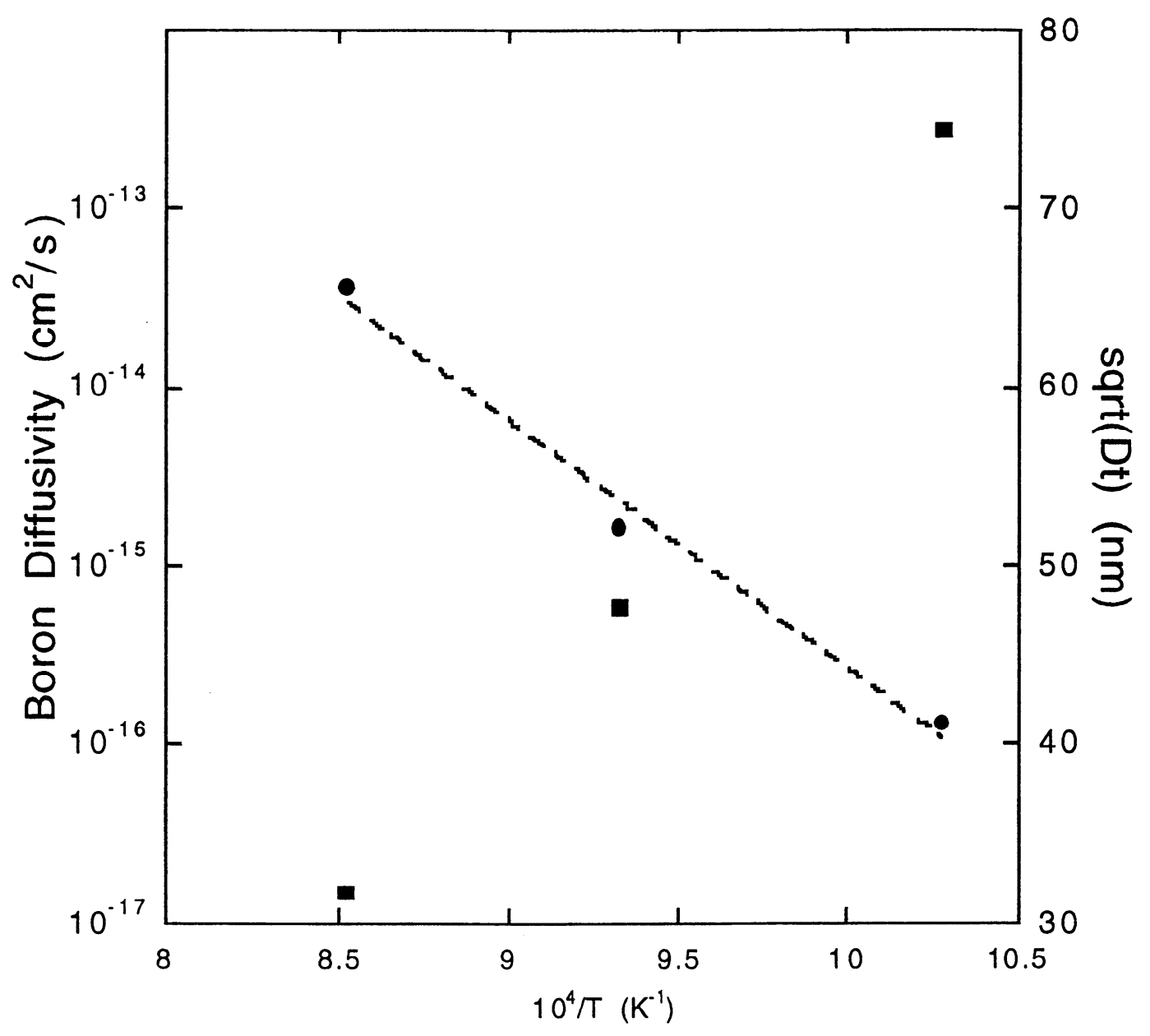




\section{FIGURE 6(a)}

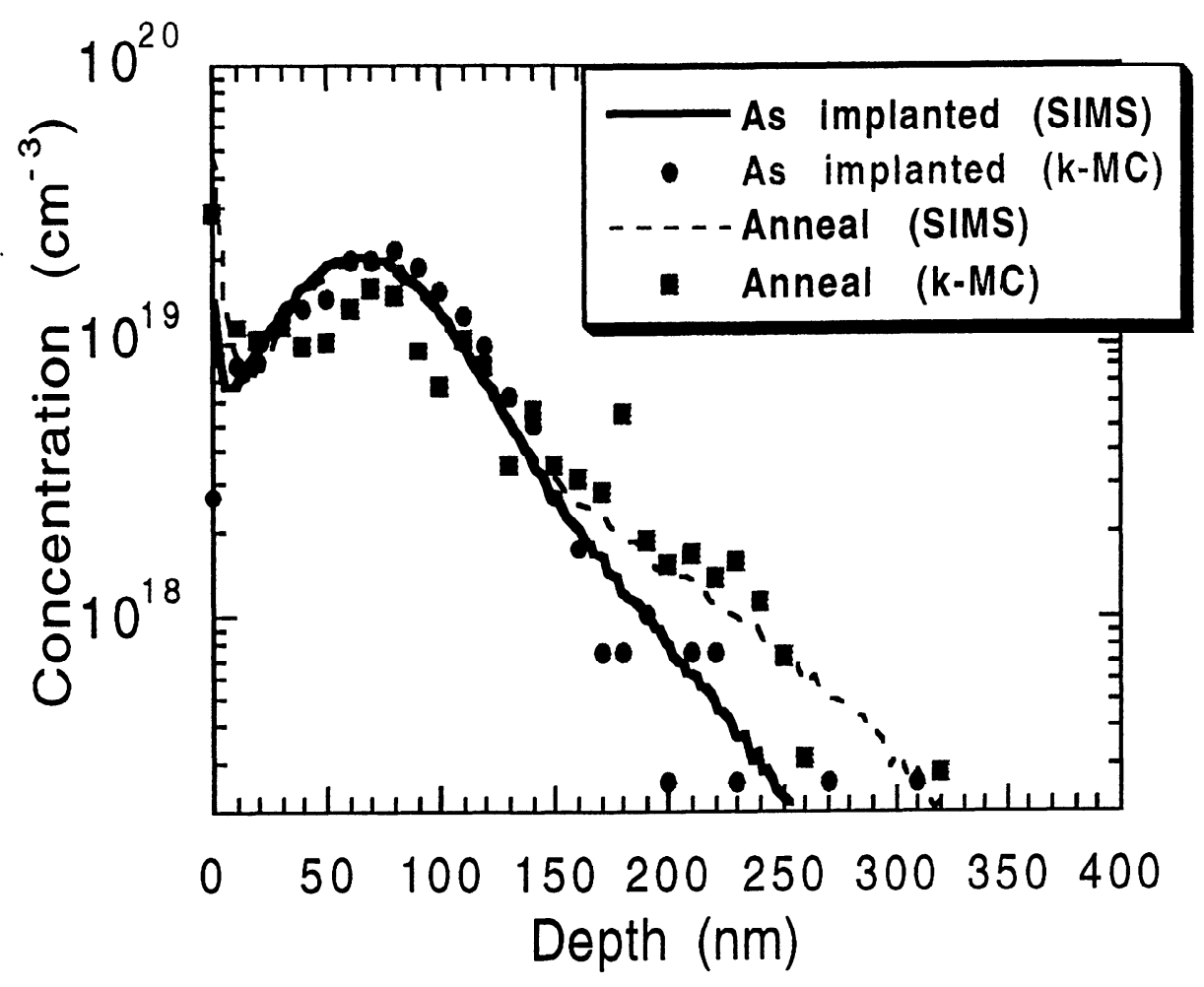


FIGURE 6(b)

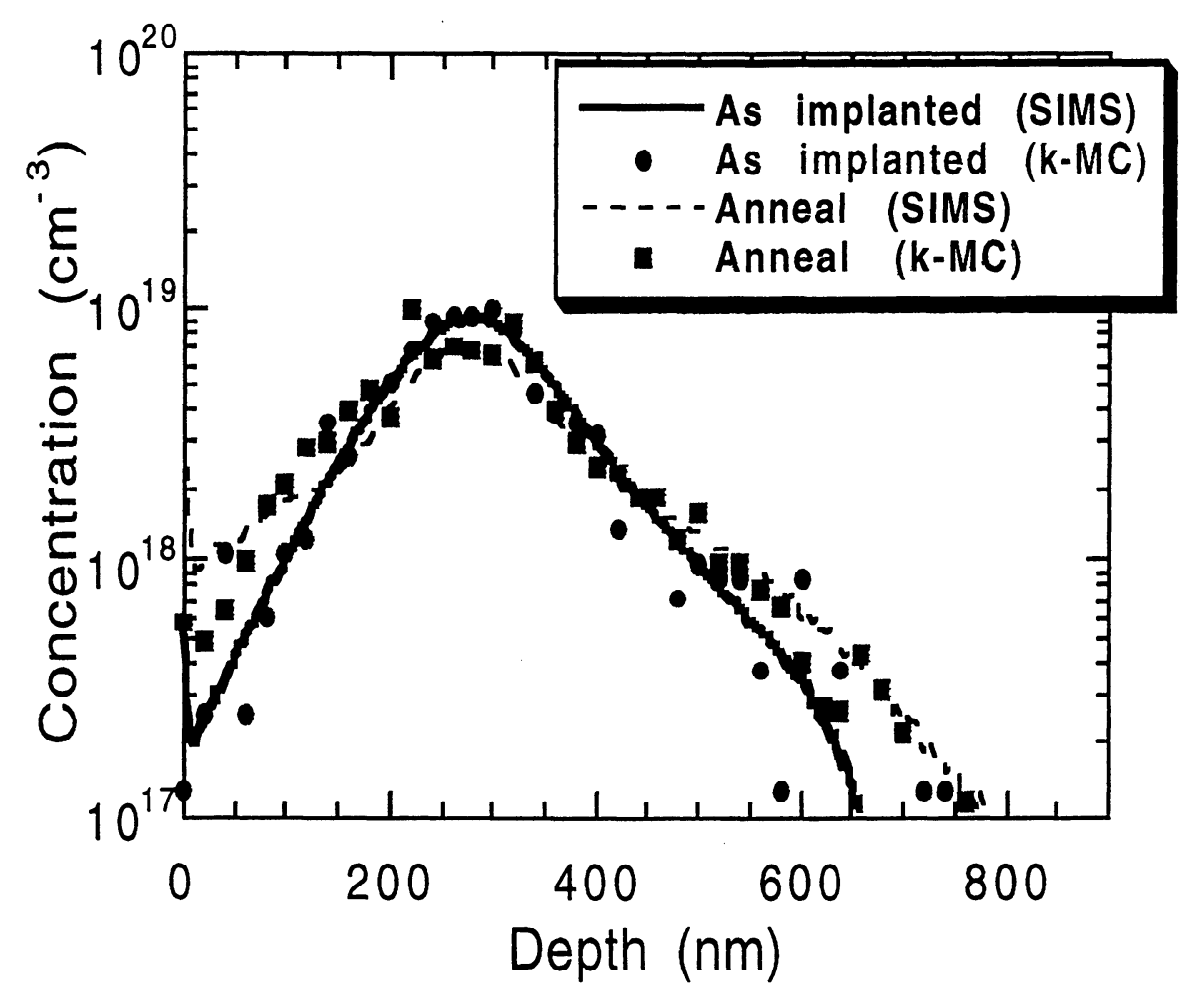




\section{FIGURE 7}

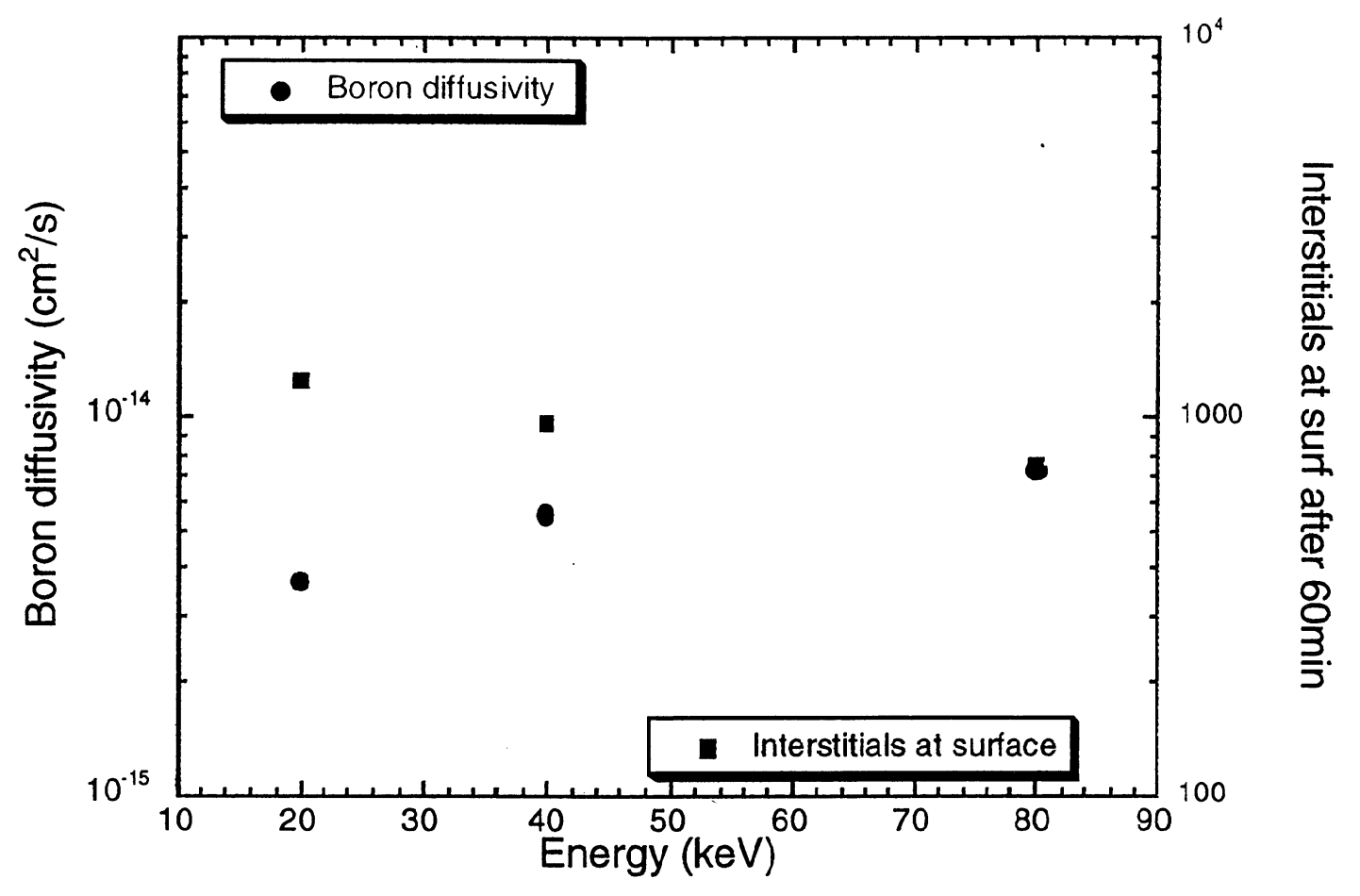


FIGURE 8

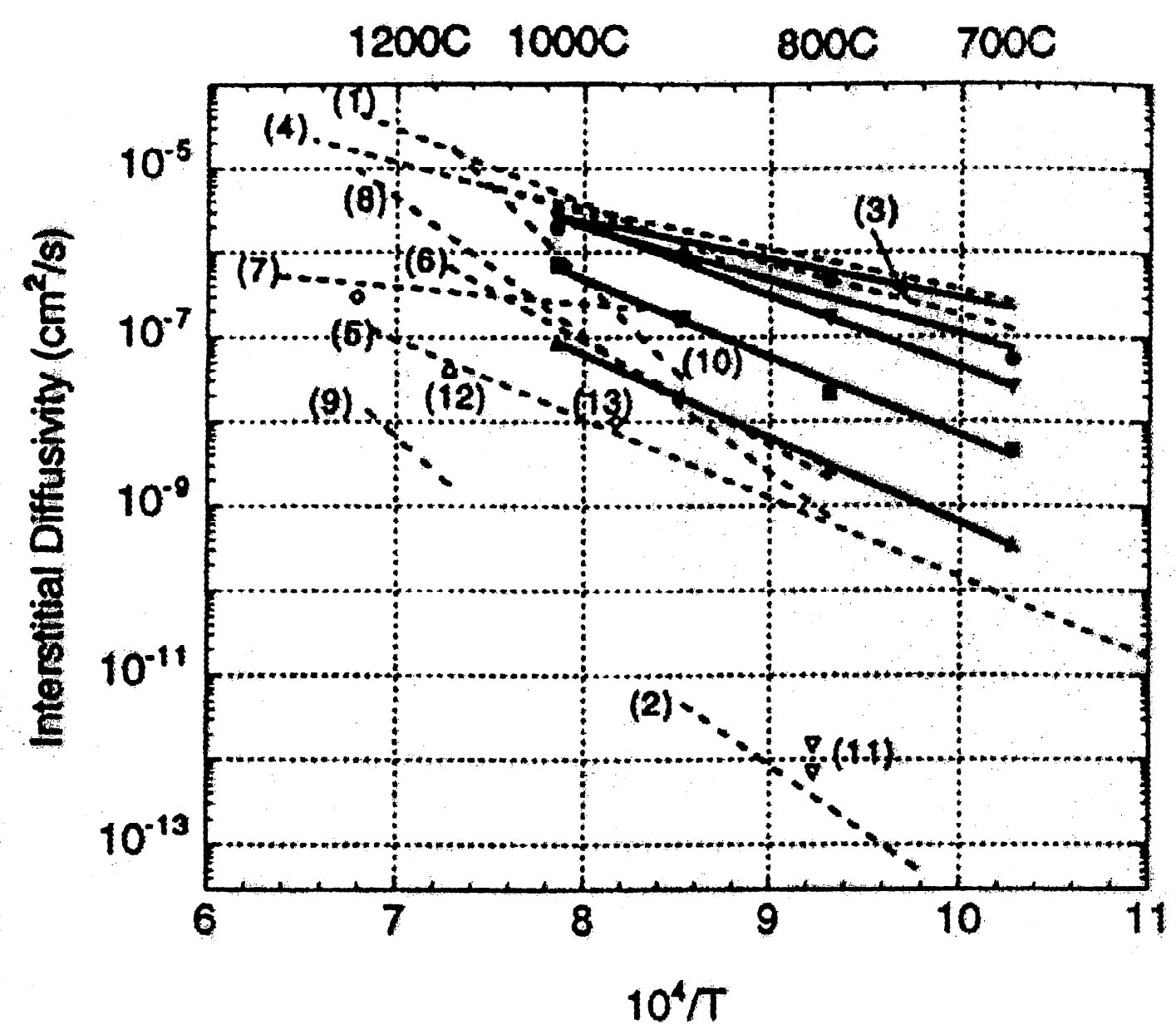




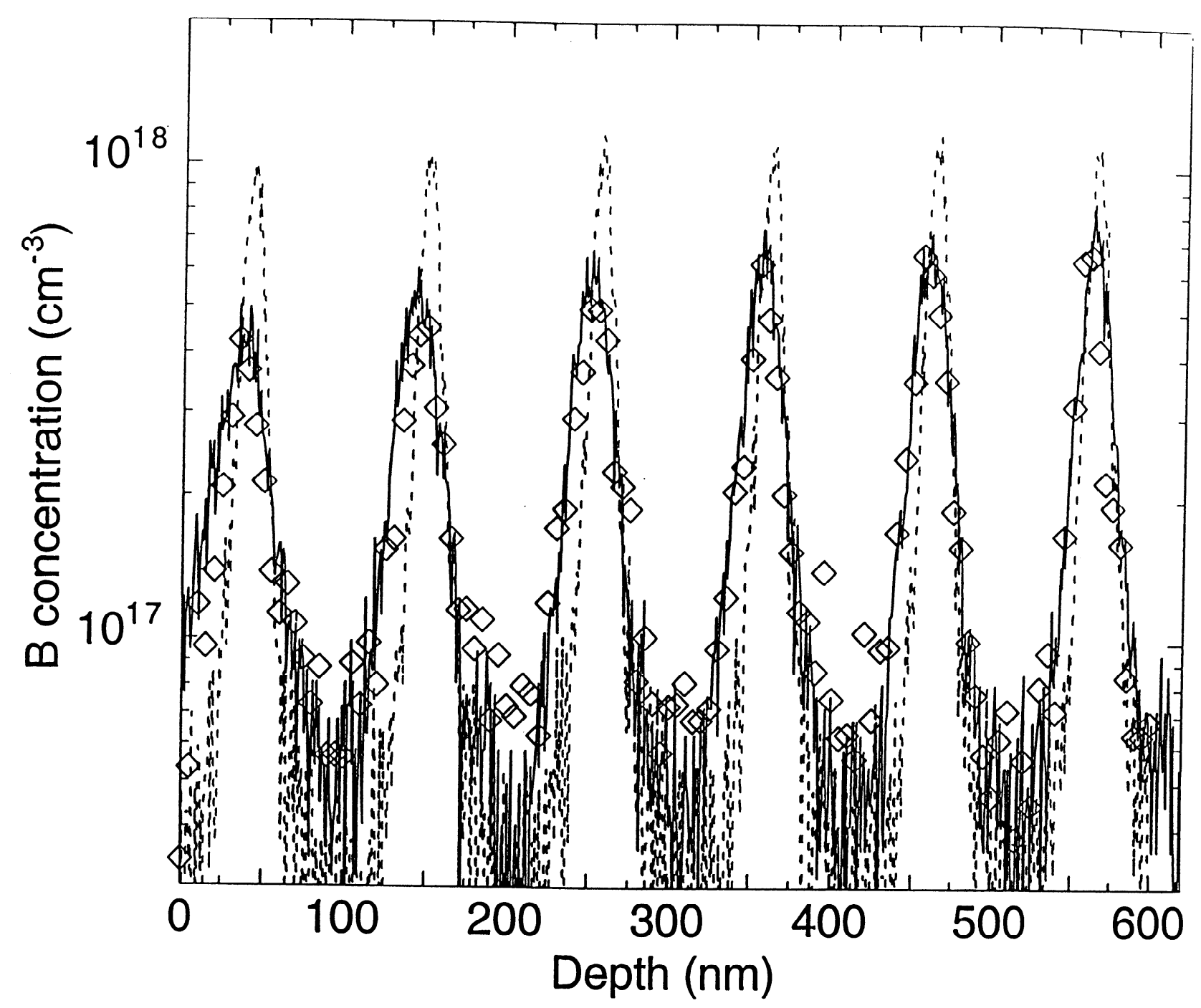

Tigure 9 


\section{FIGURE 10}

Comparison of Our Model to KM C and Experiment

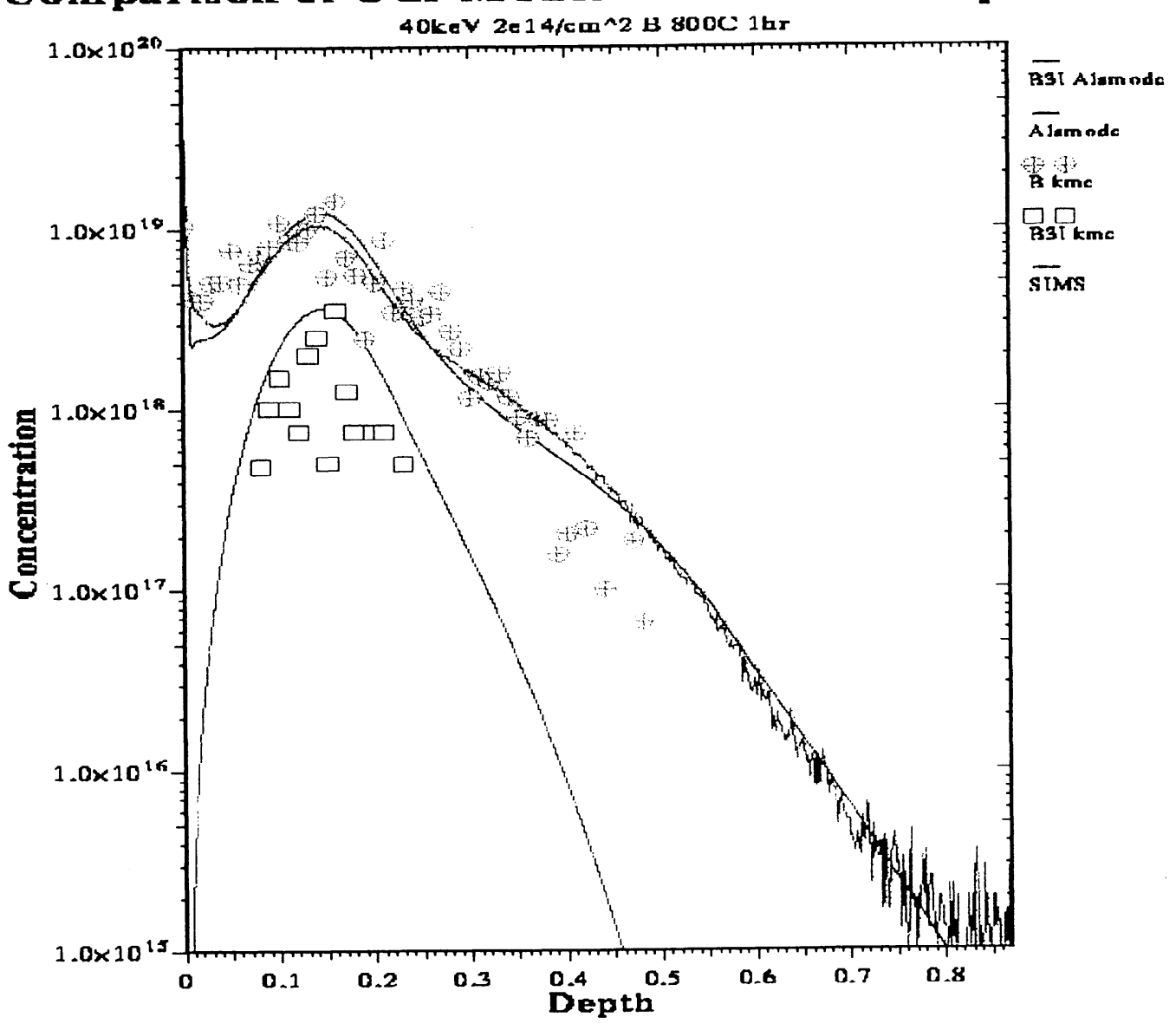


